\title{
A Low Complexity Pilot Scheduling Algorithm for Massive MIMO
}

\author{
Jung-Chieh Chen, Member, IEEE, Chao-Kai Wen, Member, IEEE, Shi Jin, and Kai-Kit Wong, Fellow, IEEE
}

\begin{abstract}
Pilot contamination is a fundamental bottleneck in massive multiple-input multiple-output (MIMO) cellular networks. In this letter, we aim to design a pilot scheduling method to reduce the effect of pilot contamination in multi-user multicell massive MIMO systems. Mathematically, the pilot scheduling problem can be formulated as a permutation-based optimization problem. However, finding the optimal solution requires an exhaustive search and is computationally prohibitive. Therefore, we propose a low-complexity near-optimal algorithm developed from the cross-entropy optimization (CEO) framework to solve this problem. Simulation results reveal that our algorithm not only significantly outperforms the existing pilot-scheduling schemes but also achieves excellent performance with low complexity.
\end{abstract}

Index Terms-Cross-entropy optimization, interference, massive MIMO, pilot contamination, pilot scheduling.

\section{INTRODUCTION}

$\mathbf{T}$ HE PIONEERING work by Marzetta in [1] has demonstrated that employing massive multiple-input multipleoutput (MIMO) techniques with time-division duplex (TDD) operation mode promises to enhance greatly the performance of cellular networks. Under the assumption of unlimited numbers of base station (BS) antennas, the effects of uncorrelated noise as well as intracell interference can be removed by the virtue of the law of large numbers [1]. The only impairment that cannot be eliminated in the asymptotic regime is the inter-cell interference caused by reusing orthogonal pilots in adjacent cells [1]. This phenomenon is commonly referred to as "pilot contamination", which restrains the achievable signal-to-interference-plus-noise ratio (SINR). Considering the channel coherence time is rather short, the number of available orthogonal pilot sequences is thus limited. Therefore, pilot reuse across cells is inevitable when the number of cells and the number of UTs per cell increase. In this case, the effect of pilot contamination must be reduced to facilitate practical implementation of multi-cell massive MIMO systems.

Several approaches have been proposed to mitigate the pilot contamination effect in multi-cell massive MIMO systems [25]. The work in [2] proposes an intra-cell pilot reuse scheme by leveraging spatially correlated Rayleigh fading channels. The solution presented in [3] proposes a joint design of channel estimation and pilot assignment method by exploiting the channel sparsity to reduce pilot contamination. In [4], a pilot allocation scheme that uses graph coloring algorithm (GCA) is proposed to mitigate pilot contamination. Although GCA performs close to the optimal exhaustive search algorithm

Manuscript received October 1, 2016

J.-C. Chen is with the Department of Optoelectronics and Communication Engineering, National Kaohsiung Normal University, Kaohsiung 802, Taiwan (e-mail: jcchen@nknu.edu.tw).

C.-K. Wen is with the Institute of Communications Engineering, National Sun Yat-sen University, Kaohsiung 804, Taiwan.

S. Jin is with the National Mobile Communications Research Laboratory, Southeast University, Nanjing 210096, P. R. China.

$\mathrm{K} . \mathrm{K}$. Wong is with the Department of Electronic and Electrical Engineering, University College London, United Kingdom.
(ESA), the required complexity remains high. ${ }^{1}$ The work in [5] develops low-complexity pilot scheduling schemes based on the tabu search (TS) algorithm and its variant to suppress pilot contamination. However, there is a performance gap between the TS-based algorithms and ESA. Moreover, the TS-based algorithms are highly sensitive to the initial guess values and easily get stuck in local optima. As a result, the performance gap between the TS-based algorithms and ESA cannot be narrowed by directly increasing the complexity (the number of iterations) of the TS-based algorithms.

To achieve near-optimal performance with consideration of the required computational complexity, a new method based on the cross-entropy optimization (CEO) framework [6] is proposed to reduce both the pilot contamination effect and the computational load. The key results and our contributions are twofold. First, we define a parameterized family of sampling distributions based on the scenario in which the same set of pilot sequences is reused in every cell, to specify how the candidate pilot assignments are generated. Then we derive an updating rule that minimizes the Kullback-Leibler distance between the parameterized distribution and the optimal importance sampling distribution. Designing an appropriate sampling distribution for the pilot scheduling problem and the corresponding update rule, which are non-trivial, are critical for good performance. Second, simulation results demonstrate that the average achievable rate per UT obtained by the proposed algorithm converges to within $99.9 \%$ of the optimal result implemented by ESA at much lower complexity.

\section{System Model AND Problem Formulation}

We consider a TDD-based multi-cell massive MIMO system with $Q$ cells, where each cell has one BS with $M$ antennas and $K(K \ll M)$ uniformly distributed active UTs with a single antenna. For clarity, the $k$-th UT in the $p$-th cell is referred to as UT $\langle p, k\rangle$. Then the channel gain vector $\mathbf{g}_{\langle p, k\rangle, q} \in \mathbb{C}^{M \times 1}$ coupling UT $\langle p, k\rangle$ to the $q$-th BS can be written as [1]

$$
\mathbf{g}_{\langle p, k\rangle, q}=\sqrt{\beta_{\langle p, k\rangle, q}} \mathbf{h}_{\langle p, k\rangle, q}
$$

where $\mathbf{h}_{\langle p, k\rangle, q} \in \mathbb{C}^{M \times 1}$ and $\beta_{\langle p, k\rangle, q} \in \mathbb{R}$ are the small-scale fading vector and the large-scale channel coefficient for the channel between the UT $\langle p, k\rangle$ and the $q$-th $\mathrm{BS}$, respectively. Here, the entries of $\mathbf{h}_{\langle p, k\rangle, q}$ are modeled as independent and identically distributed (i.i.d.) zero-mean circularly-symmetric complex Gaussian random variables with unit variance, and $\beta_{\langle p, k\rangle, q}$ is modeled as [1]

$$
\beta_{\langle p, k\rangle, q}=\frac{s_{\langle p, k\rangle, q}}{d_{\langle p, k\rangle, q}^{\alpha}},
$$

${ }^{1}$ According to the report of [4], when both the number of cells and number of UTs per cell are 4 , ESA requires $(4 !)^{3}=13,824$ trials to achieve the optimal pilot assignment, whereas $20 \times 2 \times 4^{2} \times 4^{2}=10,240$ trials are required for GCA to obtain the desired pilot assignment. Consequently, GCA needs approximately $74.07 \%$ of the computational complexity of ESA 
where $d_{\langle p, k\rangle, q}$ is the distance between UT $\langle p, k\rangle$ and the $q$-th BS, $\alpha$ is the propagation exponent, and $s_{\langle p, k\rangle, q}$ is a shadowing lognormal term with a standard deviation of $\sigma_{\text {shadow }} \mathrm{dB}$.

In a TDD network, the channel state information at BSs can be acquired by pilot-aided channel estimation. However, considering the limited number of orthogonal pilot sequences, we assume that the same set of orthogonal pilot sequences with length $\tau$ represented by $\boldsymbol{\Phi}$ is reused in every cell. Here, $\boldsymbol{\Phi}=\left[\phi_{1} \phi_{2} \cdots \phi_{K}\right] \in \mathbb{C}^{\tau \times K}$ denotes the pilot matrix with orthonormal column vectors such that $\boldsymbol{\Phi}^{\mathrm{H}} \boldsymbol{\Phi}=\mathbf{I}_{K}{ }^{2}$ To assign the pilot for each UT, we introduce a variable $a_{p, k} \in\{1,2, \ldots, K\}$ to denote that the pilot sequence $\phi_{a_{p, k}}$ is assigned to UT $\langle p, k\rangle$. An intuitive pilot assignment approach is to randomly allocate the pilot sequence $\phi_{a_{p, k}}$ to $\mathrm{UT}\langle p, k\rangle$ while ensuring that every pilot is not reused within the same cell. Thus, the pilot assignment user index matrix $\mathbf{A}$ for the entire system can be described as $\mathbf{A}=\left[a_{q, k}\right] \in \mathbb{N}^{Q \times K}$.

Given a pilot assignment user index matrix $\mathbf{A}$, the uplink SINR of UT $\langle p, k\rangle$ is given by $[1,4,7]$

$$
\begin{aligned}
\operatorname{SINR}_{\langle p, k\rangle}^{u l}(\mathbf{A}) & =\frac{\left|\mathbf{g}_{\langle p, k\rangle, p}^{\mathrm{H}} \mathbf{g}_{\langle p, k\rangle, p}\right|^{2}}{\sum_{\langle q, l\rangle \in \mathcal{S}_{\langle p, k\rangle}}\left|\mathbf{g}_{\langle q, l\rangle, p}^{\mathrm{H}} \mathbf{g}_{\langle q, l\rangle, p}\right|^{2}+\sigma_{\langle p, k\rangle}^{2}} \\
& \rightarrow \frac{\beta_{\langle p, k\rangle, p}^{2}}{\sum_{\langle q, l\rangle \in \mathcal{S}_{\langle p, k\rangle}} \beta_{\langle q, l\rangle, p}^{2}}, \text { as } M \rightarrow \infty,
\end{aligned}
$$

where $\sigma_{\langle p, k\rangle}^{2}$ is the power of uncorrelated interference and noise, $\mathcal{S}_{\langle p, k\rangle}=\left\{\langle q, l\rangle: a_{q, l}=a_{p, k}\right\} \backslash\{\langle p, k\rangle\}$ denotes the set of UTs in different cells using the same pilot sequence as UT $\langle p, k\rangle$, and the denominator of the right side of (4) is called pilot contamination. Accordingly, the ergodic achievable rate of $\mathrm{UT}\langle p, k\rangle$ in the uplink can be calculated as $[4,7]$

$$
\mathcal{C}_{\langle p, k\rangle}^{\mathrm{ul}}=\left(1-\mu_{0}\right) \mathbb{E}\left\{\log _{2}\left(1+\operatorname{SINR}_{\langle p, k\rangle}^{\mathrm{ul}}\right)\right\},
$$

where $\mu_{0}$ is the fraction of time slots which are allocated to channel estimation $[1,4]$, and the expectation $\mathbb{E}$ is taken with respect to the small-scale fading channels. In this work, our goal is to find an optimal $\mathbf{A}^{\star}$ that maximizes the total system throughput, subject to the constraint that every pilot cannot be reused within one cell. Thus, we have the following pilot scheduling optimization problem:

$$
(\mathcal{P} 1): \max _{\mathbf{A}} \sum_{p=1}^{Q} \sum_{k=1}^{K} \mathbb{E}\left\{\log _{2}\left(1+\operatorname{SINR}_{\langle p, k\rangle}^{\mathrm{ul}}(\mathbf{A})\right)\right\} .
$$

Unfortunately, problem $\mathcal{P} 1$ cannot be solved because it requires accurate channel state information that is unavailable under pilot contamination. However, the large-scale channel coefficients $\beta_{\langle p, k\rangle, q}$ change slowly over time and can be easily tracked by the BSs. Consequently, problem $\mathcal{P} 1$ can be approached by solving the following solvable pilot scheduling optimization problem:

$$
(\mathcal{P} 2): \max _{\mathbf{A}} \mathcal{R}(\mathbf{A})
$$

\footnotetext{
${ }^{2}$ We consider that each cell has the same number of UTs and the number of pilots equals the number of UTs for ease of notation. However, the proposed scheme can work under more relaxed system configurations.
}

where

$$
\mathcal{R}(\mathbf{A})=\sum_{p=1}^{Q} \sum_{k=1}^{K} \log _{2}\left(1+\frac{\beta_{\langle p, k\rangle, p}^{2}}{\sum_{\langle q, l\rangle \in \mathcal{S}_{\langle p, k\rangle}} \beta_{\langle q, l\rangle, p}^{2}}\right) .
$$

We can now see that $\mathcal{P} 2$ is a permutation-based optimization problem, and its optimal solution can be obtained using the ESA. However, the computational complexity for performing ESA to find $\mathbf{A}^{\star}$ is $(K !)^{Q-1}$, which unfortunately incurs prohibitively high computational complexity.

\section{Proposed Algorithm}

\section{A. CEO Framework Overview}

Methodologically, CEO is a population model-based optimization algorithm that probabilistically generates a population of candidate solutions from a parameterized probability distribution over the solution space $\mathcal{X}$. The core idea of CEO is to iteratively update the parameters of the distribution based on the generated candidate solutions to guide future search toward promising regions that consist of highly fit solutions. To this end, let $\mathscr{L}(x ; v)$ be a probability distribution of a random variable $X$ with parameter $v$ and let $\mathrm{C}(\cdot)$ be a real-valued cost function defined on $\mathcal{X}$. We aim to find the optimal solution $X^{\star}$ that maximizes $\mathrm{C}(\cdot)$ over $\mathcal{X}$ by means of CEO.

Starting from an initial reference parameter $v \triangleq v_{\text {initial }}$, each iteration of CEO to solve an optimization problem involves the following three steps. First, a random set of $S$ candidate solutions, denoted by $\left\{X_{s}\right\}_{s=1}^{S} \in \mathcal{X}$, are drawn from the parameterized distribution $\mathscr{L}(\cdot ; v)$. Then all the candidate solutions are evaluated to produce a set of performance values $\left\{\mathrm{C}\left(X_{s}\right)\right\}_{s=1}^{S}$. Second, the top- $\lceil\varrho S\rceil$ performing candidate solutions (called "elite samples") are selected, where $\varrho \in(0,1)$ denotes the quantile coefficient (called "elite ratio") and $\lceil\cdot\rceil$ is the ceiling operation. Here, the worst performance of the elite samples is denoted by $\eta$. Third, the parameter $v$ is updated based only on the elite samples to produce an improved solution set in the next iteration. To be precise, this update process is conducted by minimizing the Kullback-Leibler distance (or cross-entropy) between the optimal distribution that concentrates on the promising solution set $\left\{X_{s}: \mathcal{C}\left(X_{s}\right) \geq \eta\right\}$ and the parameterized distribution $\mathscr{L}(\cdot ; v)$. According to [6], this process is equivalent to solving

$$
\max _{v} \frac{1}{S} \sum_{s=1}^{S} \mathbb{1}_{\left\{\mathcal{C}\left(X_{s}\right) \geq \eta\right\}} \ln \mathscr{L}\left(X_{s} ; v\right),
$$

where the indicator function $\mathbb{1}_{\text {\{condition }}$ is " 1 " if the condition is true and " 0 " otherwise. Through an iterative update of the parameter $v, \mathscr{L}(\cdot ; v)$ eventually converges to the optimal importance sampling distribution, which concentrates all its mass at the point $X^{\star}$.

\section{B. CEO for the Pilot Scheduling Problem}

To develop the CEO framework for the pilot scheduling problem (7), we first need to establish a random mechanism to generate a pilot assignment user index matrix $\mathbf{A}$. Since there are $Q$ cells and $K$ pilots that can be reused in every cell, we 
define $Q$ auxiliary $K \times K$ probability matrices $\boldsymbol{\pi} \triangleq\left\{\mathbf{V}^{q}=\right.$ $\left.\left[v_{k l}^{q}\right]\right\}_{q=1}^{Q}$, where $v_{k l}^{q}$ is the probability of allocating the $l$-th pilot sequence to UT $\langle q, k\rangle$. Thus, the probability distribution $\mathscr{P}(\cdot ; \boldsymbol{\pi})$ of $\mathbf{A}$ is characterized by $\boldsymbol{\pi}$ and defined as

$$
\mathscr{P}\left(\mathbf{A}_{s} ; \boldsymbol{\pi}\right) \triangleq \prod_{q=1}^{Q} \prod_{k=1}^{K} \prod_{l=1}^{K}\left(v_{k l}^{q}\right)^{\mathbb{1}_{k l}^{q}\left(\mathbf{A}_{s}\right)}
$$

where $\mathbb{1}_{k l}^{q}\left(\mathbf{A}_{s}\right)$ is an indicator variable, which yields " 1 " when the $(q, k)$-th element of $\mathbf{A}_{s}$ is $l$, and " 0 " otherwise. Note that $\mathbf{A}$ is a random matrix with probability density function $\mathscr{P}(\cdot ; \boldsymbol{\pi})$, whereas $\mathbf{A}_{s}$ is a random sample (candidate solution) drawn from $\mathscr{P}(\cdot ; \boldsymbol{\pi})$.

Once the parameterized distribution is determined, we have to specify the updating rule for the parameters $\pi=\left\{\mathbf{V}^{q}=\right.$ $\left.\left[v_{k l}^{q}\right]\right\}_{q=1}^{Q}$ based on the Kullback-Leibler distance minimization program (9). To do this, a set of $S$ candidate pilot assignment user index matrices $\left\{\mathbf{A}_{s}\right\}_{s=1}^{S}$ are initially drawn from distribution $\mathscr{P}(\cdot ; \boldsymbol{\pi})$ defined in $(10)$. Then we test the performance of each candidate solution via (8) to obtain a set of performance values $\left\{\mathcal{R}\left(\mathbf{A}_{s}\right)\right\}_{s=1}^{S}$. However, different from (9), we also require the elements in each column of $\mathbf{V}^{q}$ to amount to 1 for the considered problem because the sum total probability of a pilot sequence being allocated to any user within one cell is obviously 1 . In this case, the optimal update problem in (9) becomes

$$
\begin{aligned}
\max _{\boldsymbol{\pi}} & \frac{1}{S} \sum_{s=1}^{S} \mathbb{1}_{\left\{\mathcal{R}\left(\mathbf{A}_{s}\right) \geq \eta\right\}} \ln \mathscr{P}\left(\mathbf{A}_{s} ; \boldsymbol{\pi}\right) \\
\text { subject to } & \sum_{k=1}^{K} v_{k l}^{q}=1, \forall q, l .
\end{aligned}
$$

Therefore, to maximize (11) with the condition that the row of each $\mathbf{V}^{q}$ sums up to 1 , we need to solve the following maximization problem by introducing Lagrange multipliers $\left\{\lambda_{l}^{q}\right\}_{l=1, l=K}^{q=1, q=Q}$ for each column of $\mathbf{V}^{q}$ as follows:

$$
\begin{aligned}
\max _{\boldsymbol{\pi}} \frac{1}{S} \sum_{s=1}^{S} \mathbb{1}_{\left\{\mathcal{R}\left(\mathbf{A}_{s}\right) \geq \eta\right\}} & \ln \mathscr{P}\left(\mathbf{A}_{s} ; \boldsymbol{\pi}\right) \\
& +\sum_{q=1}^{Q} \sum_{l=1}^{K} \lambda_{l}^{q}\left(\sum_{k=1}^{K} v_{k l}^{q}-1\right) .
\end{aligned}
$$

By following the first-order conditions to find the optimum, we set the partial derivative of (12) to zero with respect to $v_{k l}^{q}$, which yields

$$
\frac{1}{S} \sum_{s=1}^{S} \mathbb{1}_{\left\{\mathcal{R}\left(\mathbf{A}_{s}\right) \geq \eta\right\}} \mathbb{1}_{k l}^{q}\left(\mathbf{A}_{s}\right)+\lambda_{l}^{q} v_{k l}^{q}=0 .
$$

Summing (13) over $k=1,2, \ldots, K$, we obtain

$$
\lambda_{l}^{q}=-\frac{1}{S} \sum_{s=1}^{S} \mathbb{1}_{\left\{\mathcal{R}\left(\mathbf{A}_{s}\right) \geq \eta\right\}} \sum_{k=1}^{K} \mathbb{1}_{k l}^{q}\left(\mathbf{A}_{s}\right) .
$$

Substituting (14) into (13) yields

$$
v_{k l}^{q}=\frac{\sum_{s=1}^{S} \mathbb{1}_{\left\{\mathcal{R}\left(\mathbf{A}_{s}\right) \geq \eta\right\}} \mathbb{1}_{k l}^{q}\left(\mathbf{A}_{s}\right)}{\sum_{s=1}^{S} \mathbb{1}_{\left\{\mathcal{R}\left(\mathbf{A}_{s}\right) \geq \eta\right\}} \sum_{k=1}^{K} \mathbb{1}_{k l}^{q}\left(\mathbf{A}_{s}\right)} .
$$

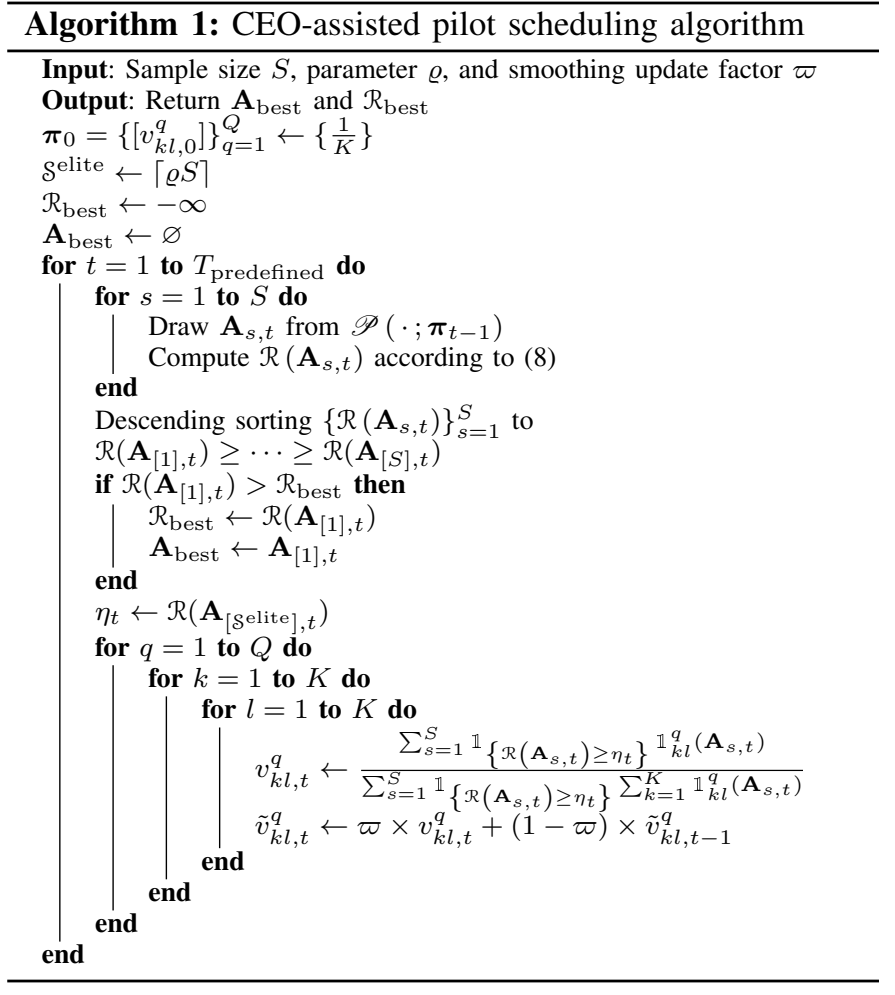

Remark 1 (Smoothed updating). Note that the updating rule in (15) is optimal for a single iteration update only. As a common practice, we employ a smooth updating procedure that computes a weighted sum of the current update and the previous update as follows [6]:

$$
\tilde{v}_{k l, t}^{q}=\varpi \times v_{k l, t}^{q}+(1-\varpi) \times \tilde{v}_{k l, t-1}^{q},
$$

where $v_{k l, t}^{q}$ is the original parameter update at the $t$-th iteration using (15), $\tilde{v}_{k l, t-1}^{q}$ is the smoothing update in the last iteration, and $\varpi \in(0,1]$ is the weight that is called the "smoothing parameter." We can observe from (16) that $\varpi$ controls the relative weight of the sample during the smoothed adaptation. If $\varpi$ is large, the convergence to a degenerate distribution may happen too rapidly and the algorithm is more likely to get stuck in the local minima. However, as pointed out in [6], the optimal solution is sampled with probability one if a proper smoothing step size is seleceted.

Remark 2 (Stopping criterion). CEO involves the iterative update of $\boldsymbol{\pi}_{t}=\left\{\left[v_{k l, t}^{q}\right]\right\}_{q=1}^{Q}$ until a predefined maximum number of iterations is reached. In this case, the complexity of CEO can be approximately analyzed in terms of the total number of samples generated, i.e., $\mathcal{O}(S \times t)$. We summarize the proposed CEO-assisted pilot scheduling algorithm in Algorithm 1.

\section{Simulation Results AND Discussion}

We consider a similar simulation setup to that in $[4,5,7]$, where the cell radius is $1,600 \mathrm{~m}$, the propagation exponent is $\alpha=3.8$, and the standard deviation of lognormal shadow fading is $\sigma_{\text {shadow }}=8 \mathrm{~dB}$. Different from that in [5], the number of BS antennas varies from $M=128$ to infinity. In addition, the loss of spectral efficiency caused by the pilot overhead is $\mu_{0}=0.2$ [4]. In addition to CEO, the classical 


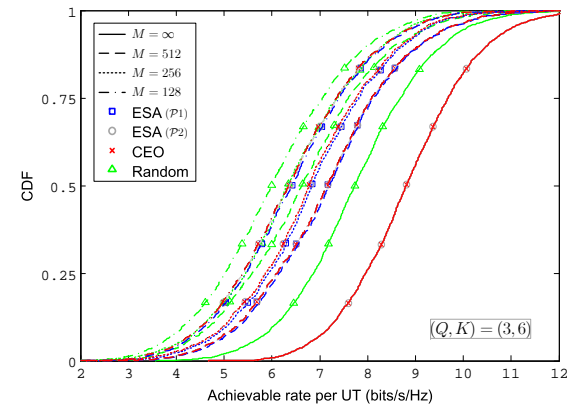

(a)

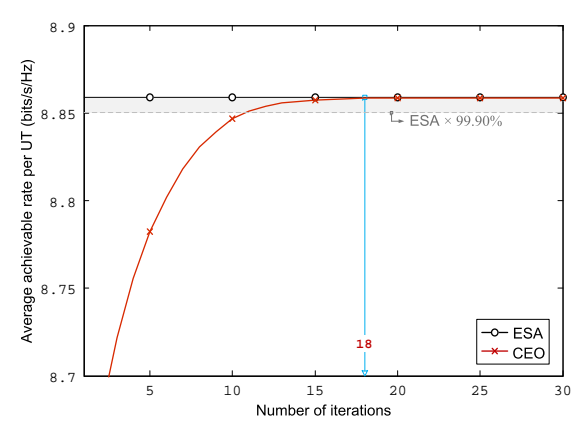

(b)

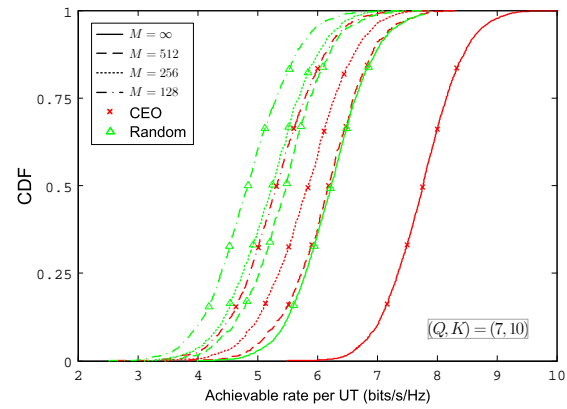

(c)

Fig. 1. Simulation results: (a) CDF of the mean achievable UL rate per UT with $(Q, K)=(3,6)$ for different algorithms. (b) Average achievable rate per UT versus the number of iterations in which the gray area indicates the range where the proposed algorithm converges to within $99.90 \%$ of the optimal result implemented by ESA. (c) CDF of the mean achievable UL rate per UT with $(Q, K)=(7,10)$ for different algorithms.

random allocation scheme [1], the ESA for $\mathcal{P} 1$, and the ESA for $\mathcal{P} 2$ are applied. Note that the ESA for $\mathcal{P} 1$ and the ESA for $\mathcal{P} 2$ produce the same results when the number of BS antennas grows to infinity, i.e., $M \rightarrow \infty$.

Fig. 1(a) shows the cumulative distribution function (CDF) curves of the achievable rate per UT of the aforementioned schemes for $(Q, K)=(3,6)$. Each $\mathrm{CDF}$ is obtained from 10,000 UT location realizations, where the ergodic rate is obtained by averaging over 10,000 small-scale fading realizations. The proposed CEO is run with $S=400, \varrho=0.1$, and $\varpi=0.2{ }^{3}$ As expected, increasing the number of BS antennas improves the achievable rate. Also, the performance gap between the ESA for $\mathcal{P} 1$ and that for $\mathcal{P} 2$ is small and becomes narrower as the number of BS antennas increases. This verifies that the solution to $\mathcal{P} 2$ under practical settings can approach the solution to $\mathcal{P} 1$ in the ideal case. In addition, enlarging the number of BS antennas widens significantly the gap between the ESA for $\mathcal{P} 2$ and the classical random allocation scheme. This illustrates that proper allocation of the pilots is necessary because the system performance significantly improves without sacrificing the multiplexing gain. Furthermore, the average achievable rate per UT obtained by CEO coincides with that by the ESA for $\mathcal{P} 2$ for the entire CDF. However, CEO requires significantly fewer computational complexities than ESA.

Fig. 1(b) depicts the average achievable rate per UT of the $\mathrm{CEO}$ for $\mathcal{P} 2$ (i.e., $M \rightarrow \infty$ ) at each iteration, averaged over 10, 000 Monte Carlo simulations, for $(Q, K)=(3,6)$, wherein the performance of the ESA for $\mathcal{P} 2$ is also included for ease of comparison. As shown in Fig. 1(b), CEO converges to within $99.99 \%$ of the true optimum obtained by ESA after approximately 18 iterations. As mentioned in Remark 2, the complexity of CEO is $\mathcal{O}\left(S \times t_{\text {conv }}\right)$, where $t_{\text {conv }}$ is the required number of iterations to reach the convergence. Therefore, CEO requires $S \times t_{\text {conv }}=400 \times 18=7,200$ trials to obtain the desired pilot assignment, whereas $(K !)^{Q-1}=518,400$ trials are required for ESA. Consequently, CEO needs approximately only $1.39 \%$ of the computational complexity of ESA.

Finally, we evaluate the performance of the proposed CEO algorithm for more complicated system configurations, i.e., $(Q, K)=(7,10)$, to evaluate its robustness. Fig. 1(c) shows

\footnotetext{
${ }^{3}$ These parameters have been selected based on the recommendation of [6] and on our own simulation experience for this problem. We have empirically determined that these parameter settings produce high-quality solutions.
}

the CDF curves of the achievable rate per UT for both the conventional random allocation and proposed CEO schemes, where CEO is run with $S=1000, \varrho=0.01, \varpi=0.2$, and $t=50$. The total number of searches required for ESA to determine the optimal pilot allocation for $(Q, K)=(7,10)$ is $2.28 \times 10^{39}$, which is too high to realize; thus, the ESA curve has been excluded in Fig. 1(c). The results show that for fixed $M$, the proposed CEO scheme significantly outperforms the conventional random allocation algorithm, which verifies the effectiveness of the proposed CEO scheme.

\section{CONCLUSION}

The pilot contamination problem can be alleviated if the UTs in neighboring cells with strong mutual interference are assigned to different pilots. However, such pilot scheduling problem is a permutation-based combinatorial optimization problem that is non-convex. In this letter, we presented an efficient iterative algorithm based on the CEO framework to tackle the pilot scheduling problem in massive MIMO cellular networks. Simulation results have verified the ability of the proposed algorithm to provide excellent performance with much reduced complexity and significant performance gain over the classical random assignment scheme.

\section{REFERENCES}

[1] T. L. Marzetta, "Noncooperative cellular wireless with unlimited numbers of base station antennas," IEEE Trans. Wireless Commun., vol. 9, no. 11, pp. 3590-3600, Nov. 2010.

[2] L. You et al., "Pilot reuse for massive MIMO transmission over spatially correlated rayleigh fading channels," IEEE Trans. Wireless Commun., vol. 14, no. 6, pp. 3352-3366, Jun. 2015.

[3] Z. Chen and C. Yang, "Pilot decontamination in wideband massive MIMO systems by exploiting channel sparsity," IEEE Trans. Wireless Commun., vol. 15, no. 7, pp. 5087-5100, Jul. 2016.

[4] X. Zhu, L. Dai, and Z. Wang, "Graph coloring based pilot allocation to mitigate pilot contamination for multi-cell massive MIMO systems," IEEE Commun. Lett., vol. 19, no. 10, pp. 1842-1845, Oct. 2015.

[5] S. Jin, M. Li, Y. Huang, Y. Du, and X. Gao, "Pilot scheduling schemes for multi-cell massive multiple-input-multiple-output transmission," IET Commun., vol. 9, no. 5, pp. 689-700, Mar. 2015.

[6] R. Y. Rubinstein and D. P. Kroese, The Cross-Entropy Method: A Unified Approach to Combinatorial Optimization, Monte-Carlo Simulation and Machine Learning. Berlin, Germany: Springer-Verlag, 2004.

[7] X. Zhu, L. Dai, Z. Wang, and X. Wang, "Weighted graph coloring based pilot decontamination for multi-cell massive MIMO systems," IEEE Trans. Veh. Technol., vol. PP, no. 99, pp. 1-1, 2016. 